\title{
REVIEW
}

\section{Study on Interface Modification and Properties of Graphene Oxide/Epoxy Composites}

\section{Yuan-Yuan Xia ${ }^{*}$ Lin Sun}

Department of Materials Processing Engineering, School of Material Science and Engineering, Liaoning Technical University, Fuxin city, Liaoning 123000, China

Corresponding Author: Yuan-Yuan Xia, Department of Materials Processing Engineering, School of Material Science and Engineering, Liaoning Technical University, No. 47 Zhonghua Rd, Fuxin city, Liaoning 123000, China. E-mail: yuanyuan_1tu@, $\underline{\text { sina.com }}$

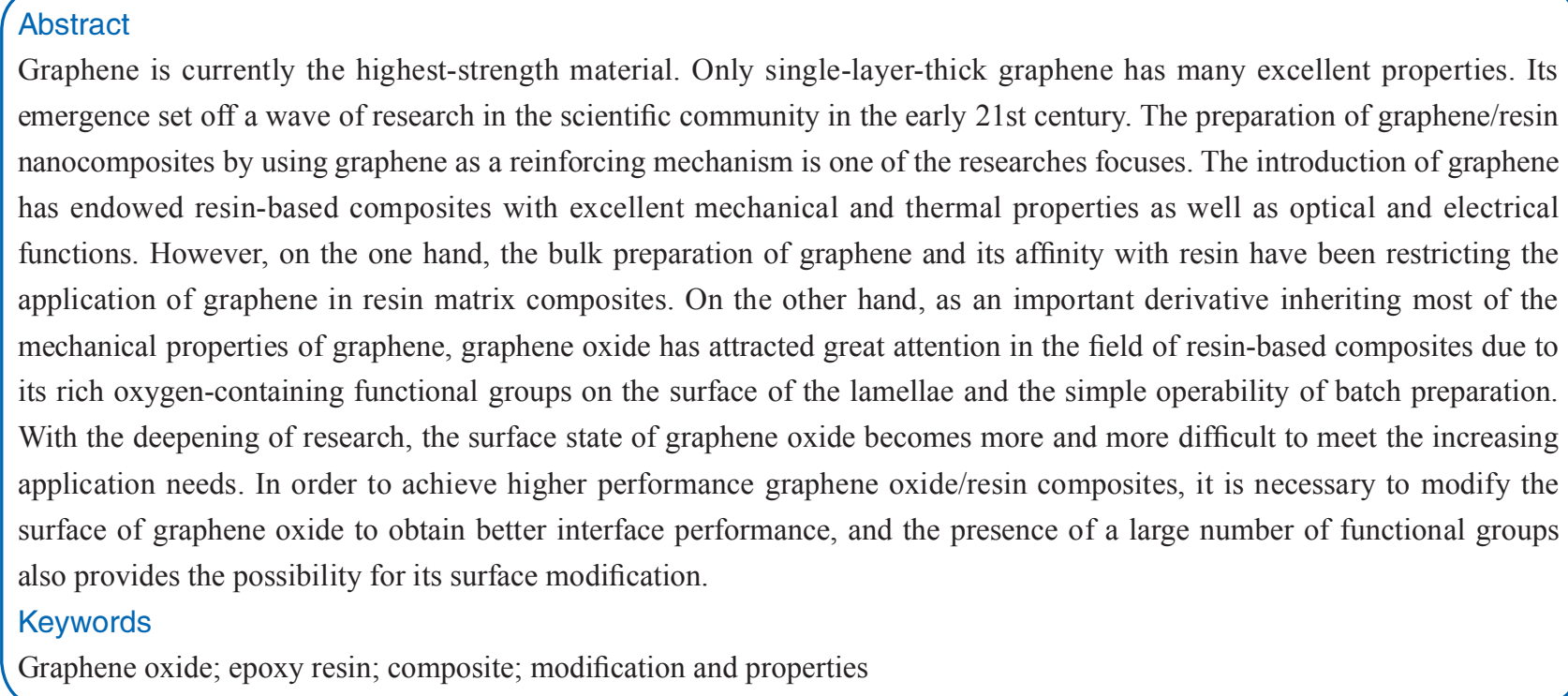

\section{INTRODUCTION}

Resin-based nanocomposites are different from traditional fiber-reinforced composites in that they are composited by a nano-scale dispersed phase with a resin matrix of less than $100 \mathrm{~nm}$. At the same time, due to the unique mechanical, thermal and photoelectric properties, large specific surface area and strong surface activity of the doped nanoparticles, these nanoparticles are dispersed in the resin matrix. Through the so-called "nanometer effect" ${ }^{\text {" }[1]}$, it can produce strong interaction with the matrix at the micro scale, so as to greatly improve the strength, heat resistance and other properties of the composite material with a

(C) The Author(s) 2019. Open Access This article is under the terms of Creative Commons Attribution 4.0 International License (https://creativecommons.org/licenses/by/4.0/), which permits unrestricted use, sharing, adaptation, distribution and reproduction in any medium or format, as long as you give appropriate credit to the original author(s) and the source, provide a link to the Creative Commons license, and indicate if changes were made. 
very small amount of addition, and give the matrix material some other new functions such as electrical properties. Therefore, resin-based nanocomposites have the characteristics of high strength, high thermal stability and excellent functionality, and are easier to process and more efficient than traditional composites. These advantages make resin-based nanocomposites show great development potential and broad application prospects ${ }^{[2]}$.

The development of nanometer reinforcement is the main factor leading the research direction of resin-based nanocomposites. The commonly used nanomaterials can be divided into polymer and inorganic nano-particles, with the latter being the main one. Among the inorganic nano-particles, in addition to the more common silica $\left(\mathrm{SiO}_{2}\right)$, titanium dioxide $(\mathrm{TiO})_{2}$ and other oxides and clay materials (such as lamellar montmorillonite, needle-stick attapulgite, etc.), the large family of carbon materials is a class of very attractive advanced nanomaterials. It covers a series of materials covering multiple scales, such as zero-dimensional fullerene (C60), one-dimensional carbon nanotubes and carbon nanofibers, two-dimensional graphene, threedimensional diamond and graphite, and has been widely studied and reported. Especially graphene, as the material with the highest strength known in nature, has both high thermal conductivity and excellent electronic transport properties. After carbon nanotubes, its appearance once again set off a wave of research on resin-based nanocomposites.

\section{GRAPHENE AND GRAPHENE OXIDE}

Graphene is a two-dimensional carbon nanomaterial with a single atomic layer thickness. Its true discovery and preparation started in 2004. Geim et al. from the school of physics at the university of Manchester, United Kingdom, obtained a perfect single-layer graphene by repeatedly taping and peeling the graphite ${ }^{[3]}$. Since graphene has excellent electrical, mechanical and thermal properties, its appearance in the field of nanomaterials has caused a great disturbance, which has also led to the rapid development of resin-based nanocomposites. However, it is not easy to prepare such ideal nanomaterials in large quantities to meet the needs of composite fillers and the integrity of the graphene carbon structure makes it difficult to achieve infiltration between it and the resin matrix, which greatly restricted the development of graphene in the field of resin-based composite materials and the final properties of composite materials. At the same time, another material that belongs to the carbon family that has not received much attention for years has gradually entered the researcher's field of vision, and this is graphite oxide. The rapid increase in the research interest of graphite oxide in academic fields is due to the close relationship with graphene. By oxidizing graphite, a traditional material, a large number of oxidizing functional groups can be intercalated into the graphite layers. The process is simple, inexpensive and has been used in industry for nearly half a century. The most important thing is that this low-cost graphite oxide can be easily stripped out of a single-layer carbon material closer to graphene, that is, graphene oxide, by simple ultrasonic treatment in water or a solvent.

The significance of graphene oxide is that it can prepare graphene-like materials in batches with a simple and low-cost process. The reason why such materials (including graphene oxide and its reduced derivatives) are called "graphene like materials" is that in the process of oxidation, the intervention of oxidation functional groups has caused damage to the aromatic ring structure of graphene lamellae and introduced some defects, which may lead to more or less loss of the perfect performance of graphene. Because of the presence of these oxygen-containing functional groups, another important property of graphene oxide is its chemical affinity with the resin matrix. In other words, because of the similarity of the main carbon structure, while maintaining most of the excellent physical properties of graphene, graphene oxide also has a large number of reactive functional groups that graphene does not have. The field of matrix composite materials is of great practical value.

\subsection{Structure and Properties of Graphene}

In fact, graphene has been regarded as a perfect ideal model by the academia for decades before its birth, and it has been considered impossible to exist stably for a long time. Such ultra-thin materials are extremely prone to curling and lamination and evolve into other carbon materials. Until Geim's group formally confirmed its existence experimentally ${ }^{[4]}$, thus leading the arrival of the graphene era.

Graphene belongs to a typical two-dimensional honeycomb carbon network structure. It consists of six-membered carbon rings that are closely arranged periodically in the plane, and the carbon atoms are connected by $\mathrm{sp}^{2}$ hybrid orbitals. Since graphene has only one carbon atom layer, its thickness is only about $0.5 \mathrm{~nm}$, as shown in Figure $1 \mathrm{a}$ ) and b). At the same time, graphene is considered as the basic constituent unit of other carbon materials. The schematic diagram of Figure 1 c) shows 
the structural evolution of graphene to other carbon materials. It can be rolled into a zero-dimensional fullerene C60. If rolled into a cylindrical shape, it forms a two-dimensional carbon nanotube. The layers are stacked on each other through a weak intermolecular interaction (Van der Waals force), which is a three-dimensional graphite structure.

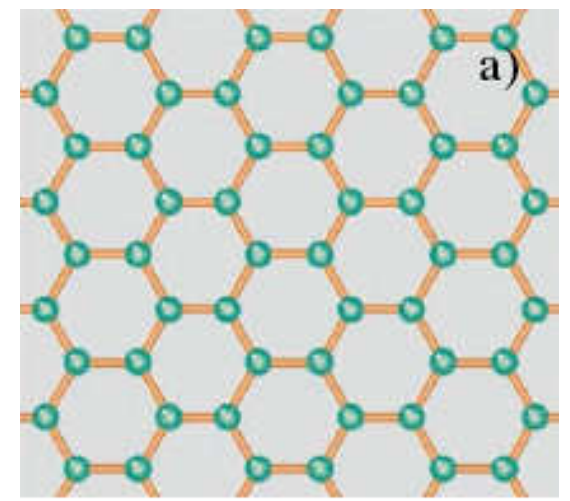

a) the carbon network of graphene

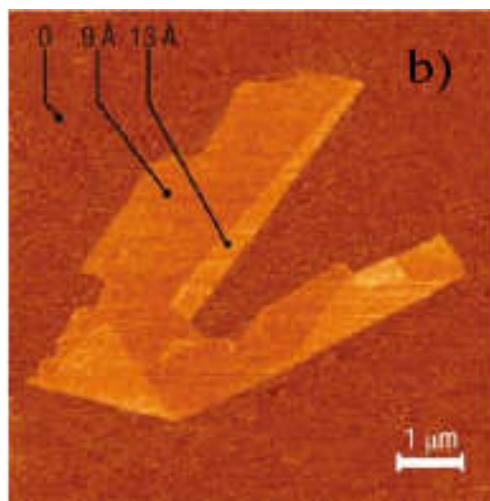

b) AFM images of graphene

c)

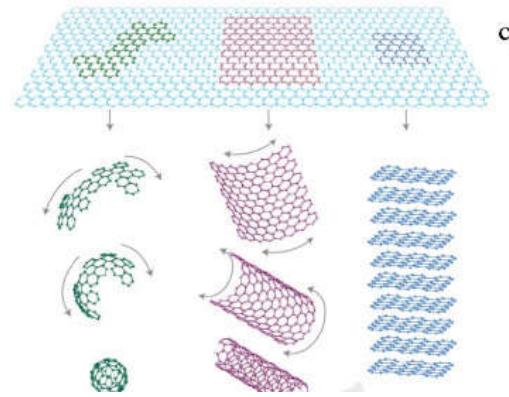

c) graphene is the parent material of all graphitic forms

Figure 1 The structure of graphene and its relationship with other carbon materials

\subsection{Structure and Properties of Graphene Oxide}

Graphene oxide, as its name implies, is composed of a large number of oxidized functional groups connected to the main structure of graphene. However, graphene oxide is also an amorphous and non-stoichiometric atomic component material, which causes great differences between individual materials. In addition to the lack of effective means of characterization, the debate over its accurate chemical structure has never stopped for years, and has not yet formed a unified conclusion ${ }^{[5]}$.

Even so, in the process of building the graphene oxide structure model, researchers have achieved great success. Earlier studies on the structure of graphene oxide believed that it was a regular lattice composed of discontinuous repeating units, and recent research results have favored non-stoichiometric models ${ }^{[6]}$. The following article will focus on two types of graphene oxide structural models, namely the Lerf-Klinowski model and the OD-bwGO model.

The structural difference between graphene and graphene oxide determines that graphene oxide cannot fully inherit the properties of graphene. The first is the difference in conductivity. The introduction of oxidized functional groups has led to the transformation of some $\mathrm{sp}^{2}$ structures of graphene to $\mathrm{sp}^{3}$ structures, which greatly reduces the conductivity of graphene oxide. Second, the presence of oxygen-containing functional groups also makes graphene oxide sacrifice heat stability. When heated to about $80{ }^{\circ} \mathrm{C}$ under air or vacuum conditions, an increase in the $\mathrm{C} / \mathrm{O}$ ratio can be clearly detected, which means that the decomposition of the oxidative functional group has occurred. Therefore, reduction of graphene oxide to repair broken carbon structures is also one of the current research hotspots in the graphene field ${ }^{[7]}$. On the other hand, the hydrophilic functional groups on the surface of graphene oxide change the hydrophobic properties of graphene and become easily dispersed in aqueous solution. Paredes et al. studied the dispersibility of graphene oxide in 13 organic solvents and concluded that graphite oxide can form a long-term stable dispersion in a variety of organic solvents and can be stripped into a single layer of oxidation in solution. Graphene undoubtedly has a positive impact on the application of graphene oxide in resin-based composites ${ }^{[8]}$. 


\subsection{Preparation of Graphene Oxide}

The most critical step in the preparation of graphene oxide is the preparation of graphite oxide. After that, a single layer of graphene oxide can be peeled off by ultrasonic oscillation in water or an organic solvent. The study of graphite oxide was accompanied by the study of graphite structure as early as 1859 years ago ${ }^{[9]}$. After more than one hundred years of development, three more representative examples of graphite oxide preparation methods have appeared, namely the Brodie method, the Staudenmaier method, and the Hummers method. Although many new preparation methods have been proposed since then, the core is still based on the improvements made by these methods, so the preparation of graphite oxide is still dominated by these three methods. The most classic and commonly used is the Hummers method, which appeared in 1958.

(1) Brodie method. Brodie, a British chemist, added potassium chlorate $\left(\mathrm{KClO}_{3}\right)$ to the slurry mixed with graphite and fuming nitric acid $\left(\mathrm{HNO}_{3}\right)$ to study the reactivity of lamellar graphite, and obtained graphite oxide with chemical elements of $\mathrm{C}, \mathrm{H}, \mathrm{O}$ and molecular formula of $\mathrm{C}_{2.19} \mathrm{H}_{0.80} \mathrm{O}_{1.00}$. He also pointed out that this kind of oxygen fossil ink is soluble in neutral and alkaline water, but not in acid medium.

(2) Staudenmaier method. Forty years later, Staudenmaier improved the preparation method of Brodie's potassium chlorate fuming nitric acid. In the oxidation process, he added potassium chlorate to the reaction system in batches instead of once adding it according to Brodie method, and added concentrated sulfuric acid to the system to increase the acidity of the mixture. The oxidation degree of graphite oxide prepared by this method is close to that of Brodie method. The C:O ratio is about 2:1, but it is more operable.

(3) Hummers method. Another 60 years later, Hummers and Offeman proposed another method to prepare graphite oxide ${ }^{[10]}$. Using potassium permanganate $\left(\mathrm{KMnO}_{4}\right)$, concentrated sulfuric acid $\left(\mathrm{H}_{2} \mathrm{SO}_{4}\right)$ and sodium nitrate $\left(\mathrm{NaNO}_{3}\right)$ to react with graphite, they also obtained graphite oxide with similar oxidation degree. This method is the most popular method for the preparation of graphite oxide.

It should be noted that the difference of reaction products of graphite oxide is very large, and even the reaction results of different batches of the same material and the same process will have slight difference of oxidation degree. This is because the product quality of graphite oxide depends not only on the oxidant, but also on the raw graphite and reaction conditions. Therefore, in the preparation process, the reaction conditions must be strictly controlled and the materials used between different batches must be unified to minimize the fluctuation of product structure and properties.

\section{PREPARATION AND CHARACTERIZATION OF GRAPHENE OXIDE AND EPOXY RESIN COMPOSITES}

The surface of graphene oxide (GO) contains a large number of oxygen-containing functional groups, and it is easy to have a certain affinity with the resin matrix. The existence of these oxygen-containing functional groups provides active sites for the design of the graphene surface chemical structure. Therefore, the preparation of graphene oxide is the starting point of this study. In this chapter, a modified Hummers method is used to prepare graphene oxide, and graphene oxide/epoxy resin composites are prepared by in-situ polymerization. As a kind of carbon material, graphene itself and its derivatives have characteristic peaks in the Raman spectrum that reflect structural characteristics. The differences and changes in these characteristic peaks can be used to study the structural differences and evolution, even the ability to monitor external loads on graphene. Raman spectroscopy will be an effective tool to help us better understand the dispersion and interfacial state of graphene in resins. Therefore, in this chapter, Raman spectroscopy will be used to study the Raman characteristics of graphene oxide and composite materials in detail, including the influence of incident laser energy, continuous irradiation time, and external load on the Raman characteristic peaks of graphene oxide.

\subsection{Preparation of Graphene Oxide and Composite Materials}

The graphene oxide was prepared using an improved Hummers method. Based on the original Hummers method, the proportions of reactants and the use of solvents were appropriately adjusted. The specific preparation process was as follows: $120 \mathrm{~mL}$ of concentrated sulfuric acid and $2.5 \mathrm{~g}$ of sodium nitrate $\left(\mathrm{NaNO}_{3}\right)$ is added to concentrated sulfuric acid. After it is dissolved, the beaker containing the reactant is placed in an ice bath and subjected to continuous magnetic stirring, and then 5 $\mathrm{g}$ of raw graphite is added. As the oxidation of graphite is a violent chemical reaction process, it will release a lot of heat in an instant, so the entire oxidation process must be kept in an ice bath. The main oxidant, potassium permanganate $\left(\mathrm{KMnO}_{4}\right)(15 \mathrm{~g})$, 
should be added one by one over several hours and the temperature of the reaction system should be controlled below $20{ }^{\circ} \mathrm{C}$ to avoid explosion caused by too fast reaction speed. After the addition of potassium permanganate, the reaction system was continuously stirred in an ice bath for $2 \mathrm{~h}$. After that, it was removed from the ice bath, and heated to $35^{\circ} \mathrm{C}$ as appropriate. The stirring was continued at this temperature for $2 \mathrm{~h}$. At this time, the reaction system was black and viscous. $250 \mathrm{~mL}$ of distilled water was slowly added. The temperature of the system increased continuously with the addition of distilled water. When the temperature rose to $90 \pm 5^{\circ} \mathrm{C}$, it was stirred at this temperature for 15 minutes, and then $500 \mathrm{~mL}$ of distilled water was added. The temperature of the system began to decrease, and it was allowed to react for $2 \mathrm{~h} .30 \mathrm{~g}$ of hydrogen peroxide solution was slowly added. With the generation of bubbles, the reaction solution gradually turns golden yellow. After stirring for 2 hours, the reaction is completed. The reaction solution was divided into six $500 \mathrm{~mL}$ centrifuge bottles and centrifuged at $5000 \mathrm{rpm}$ for $20 \mathrm{~min}$. After the supernatant was discarded, the reaction was washed repeatedly with $800 \mathrm{~mL}$ of cleaning solution (distilled water $+3 \mathrm{wt} \%$ concentrated sulfuric acid $+0.5 \mathrm{wt} \%$ hydrogen peroxide). The product was removed 10 times to remove excess potassium permanganate and oxidation product impurities, during which the solid product was extracted by centrifugation at $5000 \mathrm{rpm}$ for $20 \mathrm{~min}$. After washing with distilled water 10 times until the $\mathrm{pH}$ of the solution was 7, the solubility of graphene in the neutral aqueous solution increased, and the centrifugation rate was increased to $8000 \mathrm{rpm}$ for $30 \mathrm{~min}$. After the two-stage cleaning process was completed, the slurry-like product was dissolved in distilled water and centrifuged again at $3000 \mathrm{rpm}$ for 5 minutes at a low speed. The solid impurities deposited are discarded and the uniform dark brown go solution is retained. So far, the graphene oxide has been prepared in the form of an aqueous solution. The aqueous solution will be used in two parts. One part will be directly stored in the aqueous solution after the concentration is measured and prepared for further processing. The other part is centrifuged at $8000 \mathrm{rpm}$ for 5 minutes and the solid product is at $40{ }^{\circ} \mathrm{C}$. It was dried under vacuum for $10 \mathrm{~h}$ to obtain solid thin film-like graphene oxide.

\subsection{Effect of Post-Curing Temperature on Graphene Oxide}

A large number of research results have found that the structure of graphene oxide is sensitive to temperature due to the existence of oxidizing functional groups, and low-level damage may occur at about $80^{\circ} \mathrm{C}$. The post-curing stage of epoxy resin curing in this study needs to be maintained at $100{ }^{\circ} \mathrm{C}$ for 4 hours, which may change the structure of graphene oxide. In view of some reports, graphene oxide has been thermally reduced below $200^{\circ} \mathrm{C}$. This section will discuss the scientific issue of how the post-curing temperature of epoxy resin will affect the structure of graphene oxide.

Since the post-curing process has been initially confirmed to cause changes in graphene oxide, this study will further analyze the nature of this change. Therefore, graphene oxide was separately heat-treated at $100{ }^{\circ} \mathrm{C}$ for 4 hours to discuss the effect of curing temperature on graphene oxide. Figure 2 shows the infrared spectrum of graphene oxide before and after heat treatment. The characteristic peaks at $3420 \mathrm{~cm}^{-1}, 1728 \mathrm{~cm}^{-1}, 1626 \mathrm{~cm}^{-1}$, and $1400 \mathrm{~cm}^{-1}$ represent the hydroxyl $-\mathrm{OH}, \mathrm{C}=\mathrm{O}, \mathrm{C}=\mathrm{C}$, and $-\mathrm{COH}$ groups of graphene oxide, respectively. $1230-1285 \mathrm{~cm}^{-1}$ The absorption peak in the range of 1 represents the $-\mathrm{CO}$ group, and its intensity showed a slight decrease after heat treatment, indicating that the $100{ }^{\circ} \mathrm{C}$ heat treatment caused a small loss of the hydroxyl (-OH) and carboxyl (-COOH) groups of graphene oxide.

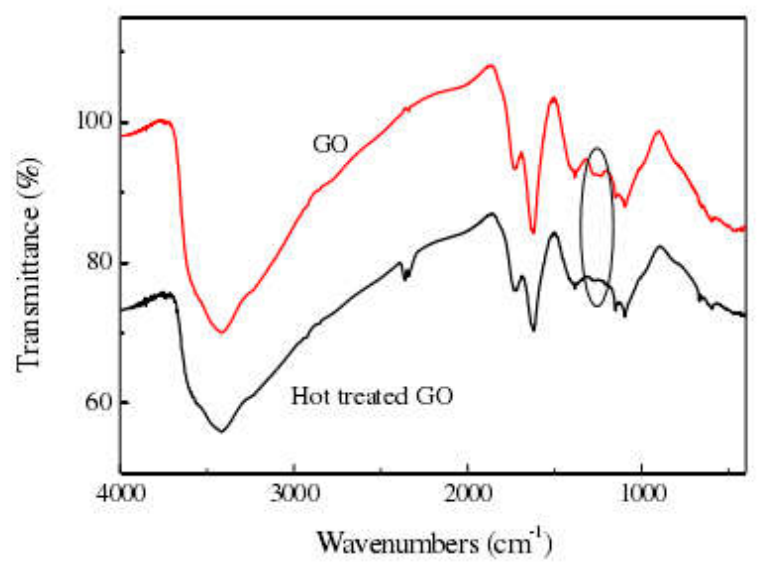

Figure 2 FTIR spectra of the graphene oxide before and after hot treatment 
The Raman characteristic peaks of go included the D peak near $1334 \mathrm{~cm}^{-1}$ and the $\mathrm{G}$ peak near $1589 \mathrm{~cm}^{-1}$. In the composite material, the characteristic summit of the epoxy matrix at $1601 \mathrm{~cm}^{-1}$ interfered with the $\mathrm{G}$ peak, but had less influence on the $\mathrm{D}$ peak. The thermal effect of the incident laser on the surface of the material by the Raman spectrometer affects the d-peak position of go. When the laser energy increases, the D-peak will move to the low wave-number position under the thermal effect. When the same level of laser energy is continuously irradiated on the sample surface, the laser with $10 \%$ energy will gradually move to the low wave-number position with the increase of irradiation time, while the laser with $1 \%$ energy will not cause significant changes. Under uniaxial strain, the d-summit of go moved to low wave number under tensile load and to high wave number under compressive load. Through this experiment, it was found that the interfacial strength of graphene oxide/epoxy composite material under tensile condition was higher than that under compression condition. In the range of $0 \%$ $0.32 \%$ strain, the interface of the composite material is relatively intact, while the increase of the strain to $0.8 \%$ will damage the interface of the two phases to some extent. It will affect the transfer of load.

\section{CONCLUSION}

The surface state of graphene oxide is a key factor that determines the properties of graphene oxide/resin composites. Five different surface states were constructed through the design and control of graphene oxide functional groups. The effects of surface state changes of graphene oxide on mechanical properties and thermal properties of graphene oxide/epoxy resin composites were studied in detail. At the same time, the Raman spectroscopy experimental method was used to reveal the microscopic mechanism of graphene oxide in the epoxy resin matrix under different surface states.

The application and research of composite nanometer reinforcement in resin matrix composites are still relatively few, but it is an effective way to obtain multi-functional composite materials. The related research in this aspect will provide a solution different from the traditional thinking for the realization of high-performance resin matrix composites.

\section{REFERENCE}

[1] Naebe M, Wang J, Amini A, Khayyam H, Hameed N, Li LH, Chen Y, Fox B. Mechanical property and structure of covalent functionalised graphene/epoxy nanocomposites. Sci Rep 2014,4:4375.

[2] Tang LC, Wan YJ, Yan D, Pei YB, Zhao L, Li YB, Wu LB, Jiang JX, Lai GQ. The effect of graphene dispersion on the mechanical properties of graphene/epoxy composites. Carbon 2013,60:16-27.

[3] Abdullah SI, Ansari MNM. Mechanical properties of graphene oxide (GO)/epoxy composites. Hbrc J 2015,11(2):151-156.

[4] Wan YJ, Tang LC, Gong LX, Yan D, Li YB, Wu LB, Jiang JX, Lai GQ. Grafting of epoxy chains onto graphene oxide for epoxy composites with improved mechanical and thermal properties. Carbon 2014,69:467-480.

[5] Jiang T, Kuila T, Kim NH, Ku BC, Lee JH. Enhanced mechanical properties of silanized silica nanoparticle attached graphene oxide/epoxy composites. Compos Sci Technol 2013,79:115-125.

[6] Li YQ, Pan DY, Chen SB, Wang QH, Pan GQ, Wang TM. In situ polymerization and mechanical, thermal properties of polyurethane/graphene oxide/epoxy nanocomposites. Mater Des 2013,47:850-856

[7] Bortz DR, Heras EG, Martin-Gullon I. Impressive fatigue life and fracture toughness improvements in graphene oxide/ epoxy composites. Macromolecules 2011,45(1):238-245.

[8] Ni Y, Chen L, Teng K, Shi J, Qian X, Xu Z, Tian X, Hu C, Ma M. Superior mechanical properties of epoxy composites reinforced by 3D interconnected graphene skeleton. ACS Appl Mater Interfaces 2015,7(21):11583-11591.

[9] Galpaya D, Wang M, George G, Motta N, Waclawik E, Yan C. Preparation of graphene oxide/epoxy nanocomposites with significantly improved mechanical properties. J Appl Phys 2014,116(5):053518.

[10] Teng CC, Ma CCM, Lu CH, Yang SY, Lee SH, Hsiao MC, Yen MY, Chiou KC, Lee TM. Thermal conductivity and structure of non-covalent functionalized graphene/epoxy composites. Carbon 2011,49(15):5107-5116. 